\title{
Acuerdo estratégico Transpacífico de Asociación Económica (TPP) y su impacto en México
}

\author{
Trans-Pacific Strategic Economic Partnership (TPP) \\ and its impact on Mexico \\ Jorge Retana Yarto*
}

Resumen

El área Asia-Pacífico cobra cada vez mayor relevancia, tanto en el comercio y las inversiones como en la investigación científica y la innovación tecnológica. Hoy, la región asiática está convertida en el gran motor y mueve los equilibrios y balances de la economía y la política mundiales de una manera importante, donde se avanza aceleradamente y los países buscan concretar acuerdos. Así, los estadounidenses han reorientado sus esfuerzos hacia un mayor control en la región Asia-Pacífico como una forma de contención del enorme poderío Chino, donde México también busca incorporarse a estos procesos de integración orientados regionalmente.

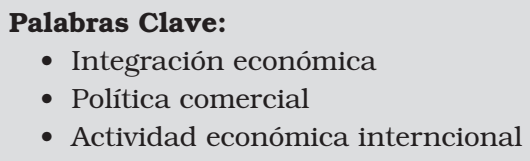

Abstract

The Asia-Pacific area is becoming increasingly important, both in trade and investment as in scientific research and technological innovation. Today, the Asian region is converted into the great engine and move the balances and balances of the economy and world politics in a major way, which progresses rapidly and countries seek to reach agreements. So, Americans have refocused their efforts towards greater control in the Asia-Pacific as a way to contain huge Chinese power, where Mexico is also seeking to join these integration processes regionally oriented.

JEL: F02, F13, F23

\section{Importancia estratégica del Área Asia-Pacífico}

Desde finales de los años ochenta del siglo pasado, se generó un amplio consenso en cuanto a que la actividad económica y los flujos de intercambio que desde la Revolución Industrial se había concentrado en el área del Océano Atlántico, en sus grandes países y ciudades se había desplazado en términos de su mayor importancia hacia el área del Océano Pacífico: tanto el comercio, como las inversiones, la investigación científica, así como la innovación tecnológica.

Este dato central, sin embargo, parecía contradecirse con un proceso de disminución en el ritmo y amplitud de la actividad económica en Japón y con la desaparición de la Unión de Repúblicas Socialistas Soviéticas (URss), que sin embargo, habían contribuido decisivamente al dinamismo económico aludido, pero que a la vez, tales pérdidas se compensaban, con la emergencia de los “Tigres Asiáticos” (Corea del Sur, Taiwán, Hong Kong, Singapur y Malasia). El proceso señalado se reafirmó con los resultados de las reformas económicas en China y la India, y la recuperación de la actividad económica a gran escala ahora de la Federación de Rusia, del Asia Central (antiguas 
repúblicas soviéticas) y con la incorporación de Hong Kong a la soberanía de China, al lado de los grandes avances económicos que reportaban todos ellos en cuanto al crecimiento del PIB, del ingreso per cápita, del ahorro, de las inversiones en el exterior, del desarrollo de sus capacidades científicas y tecnológicas. Hoy China y la India rebasan 40\% de la economía, población y territorio de Asia-Pacífico, y la Asia-Pacific Economic Cooperation (APEC) aportó 54\% del PIB mundial en 2007 (dentro de la APEC, están Estados Unidos y Japón, obviamente). ${ }^{1}$

Durante el año de 2011, conforme a los datos del Fondo Monetario Internacional (FMI) de 2012, Asia como región creció 5.8\%, en promedio, en 2012 en $5.4 \%$ y en 2013 -conforme a sus proyecciones- lo hará nuevamente retomando el porcentaje de 2011, es decir, 5.8\% del PIB promedio anual. Sus Índice de Precios al Consumidor, creció en 2011 en 5.0\%, para 2012 en $3.9 \%$ (registrando un descenso) y para 2013 se proyecta un nuevo descenso pequeño hasta una tasa de 3.8\% anual; el Saldo en Cuenta Corriente, dato fundamental de competitividad externa (en general, entre menor sea más competitiva es la economía) fue en 2011 de $1.9 \%$ respecto al PIB, será de $1.2 \%$ en 2012 y se elevará en 2013 levemente para llegar a 1.3\%, menor al año de 2011, que fue en este trienio, su tasa más alta. ${ }^{2}$

Se mantienen los ritmos de expansión muy altos de las economías de China y la India: China creció en 9.2\% durante el año de 2011, reducirá su ritmo para este año de 2012 hasta $7.8 \%$, para aumentarlo nuevamente en 2013 a 8.2\% de crecimiento anual. Su Índice de Precios al Consumidor fue de $5.4 \%$ en 2011 , será de $3.0 \%$ en 2012 y se estabilizará en el mismo nivel para 2013, 3.0\% anual; su Saldo de Cuenta Corriente fue de 2.8\% en 2011 , lo será de $2.3 \%$ en 2012 y luego se ha estimado de $2.5 \%$ en 2013 ; y su tasa anual de desempleo (con las salvedades metodológicas nacionales se ha estabilizado en $4.1 \%$ anual (por ejemplo, la mitad de Estados Unidos para 2012-2013) e inferior a México (4.8\%) para ambos años.

En el caso de India, el crecimiento de su PIB anual fue de 6.8\% para

${ }^{1}$ Para revisar los primeros materiales que se escribieron sobre el tema, ver "La Inserción de México en la Cuenca del Pacífico", Tomos I y II, Facultad de Economía, unam, 1990. Puede consultarse del autor de este ensayo: Un bloque Asiático y Latinoamericano, Opción de Cambio Estratégico para México, Montiel \& Soriano Editores, septiembre de 2011. Igualmente, Omae, Kenichi: "El próximo escenario global”, editorial Wharton School Publishing, 2005; de Engardio, Pete: "Chindia". Como China e India están revolucionando los negocios globales. Mc Graw Hill, 2008. Sobre el tema del renacimiento del poderío ruso, ver: Strategic Studies Institute Monograph ¿Can Russia Reform? Economic, Political, and Military Perspectives. Editor Stephen J. Blank, June 2012. Finalmente, la Memoria del Primer Seminario Internacional: China, América Latina y el Caribe: condiciones y retos en el siglo xxI, de muy próxima aparición, editado por el CECHIMEX-Facultad de Economía de la unAm y Unión de Universidades de América Latina (UduAL). ${ }^{2}$ Ver : "Perspectivas de la Economía Mundial", FMI, octubre de 2012, http://www.imf.org 
Economía Informa núm. 380 mayo - junio • 2013 ㅁ

2011, y se ha estimado de 4.9 y 6.0\% para los años 2012-2013; su Índice de Precios al Consumidor fue de $8.9 \%$ en 2011 , de 10.2 será para 2012 y de 9.6\%, con un leve descenso para 2013; su Saldo de Cuenta Corriente acusa signo negativo para el año de 2011 en -3.4\% como porcentaje del PIB, crecerá levemente para este año de 2012 llegando a 3.8\% anual y descenderá un poco en 2013 al ser 3.3\% en la proyección de la institución mencionada. No existen datos de su tasa anual de desempleo para estos años.

La Federación de Rusia, por su parte, con un ritmo menor, mantiene su tendencia al crecimiento en tasas aceptables y otras variables controladas, aunque mayores que los dos otros países mencionados: su crecimiento del PIB anual para 2011 fue de 4.3, 3.7 y 3.8\% como datos proyectados para el bienio 2012-2013; su Índice de Precios al Consumidor fue alto en 2011 al alcanzar 8.8\%, descenderá de manera importante en 2012 hasta 5.1\% para crecer estimada mente en 2013 a 6.6\%; y en cuanto al Saldo de la Balanza en Cuenta Corriente, sus datos son de signo positivo: de 5.3\% en 2011; de $5.2 \%$ en 22012 y descendiendo a 3.8\% 2013; mientras que su tasa anual de desempleo fue en 2011 de $6.5 \%$, descenderá y se estabilizará para 20122013 en 6.0\%, siempre conforme a Informe de FMI de octubre de 2012.

En estudio reciente de una empresa consultora internacional estadounidense (Boston Consulting Group) se establece que China e India (poco más de 3000 millones de habitantes entre ambos) triplicaran su consumo interno (por tanto, el ingreso de sus habitantes) hacia el año 2020. Serán maquinarias de producción y demanda descomunales e incontenibles en sólo 8 años más. La clase media en ambos países -para dicho año- sumará un billón de personas. Son cambios portentosos, en lo económico-social y relevantes a nivel estructural. ${ }^{3}$

Expresan el gran regreso histórico de Asia con una presencia multi-redimensionada, evocando su antiguo y gran esplendor que sucumbió ante sus propias contradicciones irresueltas, pero de igual manera, bajo el peso del colonialismo rapaz y prolongado de las potencias europeas.

En suma, la región asiática, en su inmensidad y complejidad, está convertida hoy en el gran motor de la economía y la política mundial, y a pesar de sus graves problemas geopolíticos, se ha convertido también en el gran espacio de las luchas y determinaciones estratégicas del mundo, de los equilibrios políticos y militares. Esta reconversión geo-económica y geo-estraté-

3 "China e India Triplicarán su Consumo Interno en 2020", http://www.globalasia.com, "The Dynamics of China's Next Consumption Engine", The Age of the Affluent. https://www.bcgperspectives.com 
gica de Asia ha movido los anteriores equilibrios existentes y los balances de fuerzas en la economía regional y global, lo mismo que en la política mundial. Estamos ante otra realidad planetaria que avanza aceleradamente bajo los impulsos de lo descrito. Es el cambio mayor de los últimos 150 años.

No olvidar que los propios estadounidenses han redefinido su doctrina estratégica para orientar todos sus esfuerzos hacia el avance en la región Asia Pacífico como la mejor forma de contención de la enorme influencia ganada en la magna región por China (ver Preservación del Liderazgo Global de EuA-Prioridades de Defensa en el Siglo xxI). Se trata de una realidad en expansión contundente. ${ }^{4}$

Lo anterior justifica con amplitud, que México busque insertarse en el gran movimiento de la región asiática, de sus países, de sus flujos económicos y de su influencia política, explica el intenso activismo en la incorporación a los acuerdos de integración subregional orientados a Asia-Pacífico: el "Acuerdo Comercial del Pacífico" (ACP) con Colombia, Perú y Chile, y el “Acuerdo Estratégico Transpacífico de Asociación Económica” integrado por nueve países que señalamos.

\section{Historia del acuerdo transpacífico}

En oportunidad de una reunión informal de líderes de APEC efectuada en Brunei Darussalam en octubre del año 2000, los Jefes de Estado de Chile, Nueva Zelandia y Singapur acordaron iniciar negociaciones con vistas a un acuerdo de libre comercio (P-3). La iniciativa se enmarcó dentro de las metas de APEC: liberalizar el comercio y las inversiones para el año 2010. Durante los días 17 y 18 de julio de 2003 fue realizada la primera ronda de conversaciones en Wellington, Nueva Zelandia y del 24 al 26 de septiembre se llevó a cabo la primera ronda de negociaciones técnicas en Singapur. Pero debido a las reservas planteadas por sectores agrícolas chilenos, el gobierno de este país decidió suspender la que iba a ser la segunda ronda de negociaciones. En tales circunstancias y luego de distintas consultas, el citado gobierno decidió proponer una modificación sobre la modalidad del Acuerdo, que pasaría a ser, en lugar de zona de libre comercio, un Acuerdo de Asociación Económica Estratégica (AAEE), en el que habrían de privilegiarse aspectos tales como la cooperación comercial y tecnológica. Bajo el nuevo

${ }^{4}$ García, Tobón Carlos: "China y El Giro Estratégico de Ee.uu. en Asia-Pacífico. América Latina: ¿A Dónde Va?. http://www.china-files.com/es. Ver también: "Giro Estratégico de Obama: Des -globalización Militar de EE.UU. y Contención de China”, http://vamosacambiarelmundo.org 
formato, tuvo lugar la segunda ronda de tratativas nuevamente en la ciudad de Wellington, Nueva Zelanda, los días 18 a 20 de agosto de 2004. En este encuentro, en el que participó como observadora una delegación de Brunei Darussalam, fueron tratadas diversas materias: acceso a mercados, aduanas, reglas de origen, normas técnicas, sanitarias y fitosanitarias, servicios, inversiones, propiedad intelectual, compras gubernamentales, políticas de competencia, asuntos laborales y ambientales y cooperación. El siguiente avance sustancial se registró en ocasión de la cuarta ronda, llevada a cabo en Queenstown, Nueva Zelandia, entre el 7 y 11 de marzo de 2005. La quinta ronda estuvo precedida por la incorporación de Brunei Darussalam como Estado signatario del futuro Acuerdo. En ocasión de esta quinta ronda, que culminó el 25 de abril de 2005, fueron acordados los aspectos fundamentales del Tratado. ${ }^{5}$

El Acuerdo Transpacífico de Asociación Económica (TPP siglas en inglés) también conocido como el Acuerdo- P-4, es un Tratado de Libre Comercio multilateral que tiene como objetivo liberalizar comercialmente a estas economías de la región del Asia-Pacífico. El Tratado fue firmado originalmente por Brunéi, Chile, Nueva Zelanda y Singapur, el 3 de junio de 2005 y entró en vigencia el 1 de enero de 2006. En los meses previos a su firma, se le conoció como "Acuerdo Cerrado de Asociación Económica entre Tres" (P3-CEP) que implicaba un contrato al que ningún otro país podía adherirse (los Tratados Comerciales o Acuerdos de Integración Regional se dividen en abiertos a la posterior integración de otros Estados adherentes, y en cerrados, sin posibilidad de otras adhesiones) y fue firmado inicialmente por Nueva Zelanda, Chile y Singapur, Acuerdo surgido de la Cumbre del Foro de Cooperación Asia-Pacífico realizada en Los Cabos, México en 2002, luego de cuyo convenio inicial, se realizaron (como suele ser) una serie de rondas de negociación para ir conformando cada uno de los capítulos del Acuerdo, fueron cuatro rondas en total, y en la quinta, se permitió a Brunei participar en las negociaciones (mes de abril de 2005), a partir de lo cual, se abrió el Acuerdo en curso a otros países y se le conoció en sus orígenes como Acuerdo P-4.

Es el primer acuerdo plurilateral entre países de Asia-Pacífico y América Latina. Algo realmente relevante. Si los términos negociados no son lo mejor, sino al contrario y hay aspectos muy negativos, es necesario separarlos del gran mérito anterior mencionado.

5 "Acuerdo Estratégico Transpacífico de Asociación Económica". Texto del Acuerdo. Ministerio de Relaciones Económicas de Chile, mayo de 2006. www.sice.oas.or 
Es importante destacar que el Acuerdo Estratégico Transpacífico de Asociación Económica contiene una Cláusula de Adhesión que permite que otras economías del Foro APEC se incorporen al mismo, reforzando con ello la intención original de perseguir una mayor integración y liberalización comercial en la zona Asia Pacífico. Hoy es bloque comercial abierto.

El objetivo fundamental, fue una liberalización comercial acelerada mediante la eliminación del 90\% de aranceles (impuestos al comercio exterior) para el $1^{\circ}$ de enero de 2006, y eliminarlos completamente antes de 2015 (5). Los cuatro países involucrados en el Acuerdo comparten tres características principales: son economías abiertas y dinámicas, aunque no muy grandes; siguen políticas de apertura comercial unilateral (sin esperar reciprocidad) y son miembros todos del Foro Asia-Pacífico. Posteriormente ingresaron seis países más: Australia, EuA, Malasia, Perú (se incorporó a las negociaciones el 19 de marzo de 2010) y Vietnam (pertenece también a la Asociación Económica del Sudeste Asiático, ASEAN junto con Malasia). En últimas fechas, formalmente fue aceptado México (inició negociaciones en junio de este año y fue aceptado en octubre del mismo, una vez que tuvo el respaldo de todos sus integrantes, principalmente de Estados Unidos), aunque falta la ratificación por parte del Senado de la República para darle plena validez e incorporar el Acuerdo al marco jurídico nacional. Con México el Acuerdo estaría suscrito por 10 países en total. Ha quedado pendiente de resolverse la incorporación de Canadá y Japón que también han solicitado su ingreso formal al bloque comercial. Entonces, estamos ante un acuerdo comercial de integración económica que por primera vez conjunta países considerados de diverso nivel de desarrollo, integrantes de otros bloques comerciales y de tres regiones geográficas distintas. Una gran innovación en materia de bloques comerciales.

En el marco de una reunión de líderes económicos de APEC, efectuada en Hanói, Vietnam, el Sultán de Brunei Darussalam, la Presidente de Chile, el Primer Ministro de Nueva Zelanda y el Primer Ministro de Singapur convinieron -el 20 de noviembre de 2006- que durante el segundo semestre del año 2007 serían iniciadas las negociaciones para incluir en el Acuerdo dos capítulos adicionales, uno de ellos sobre inversiones y el otro sobre servicios financieros.

Los nueve integrantes del TPP sin México, son países que participan con 30\% del Producto Interno Bruto mundial, 15\% de las exportaciones y 19\% de las importaciones mundiales, en cuyos porcentajes es determinante -en alta medida- la incidencia de Estados Unidos (23\% del PIB mundial). Las 
exportaciones de México a Asia han registrado un mayor crecimiento en los últimos cinco años con tasa de crecimiento promedio anual de $17.5 \%$. Este es un dato sólido.

Sin embargo, llama la atención que toda esta estrategia comercial de México, a diferencia de tres de sus socios comerciales latinoamericanos (Colombia, Perú y Chile, para no mencionar a los países del Mercosur) no es complementada enérgicamente fortaleciendo sus relaciones comerciales y de inversión con China, cuyo Acuerdo de Asociación Estratégica no parece desear potenciarse al máximo por México. Las bases están sentadas (hay firmado con China un Acuerdo de Asociación Estratégica desde el año 2003) pero parece que no existe la voluntad política suficiente o necesaria. ¿Se temerá irritar a Washington? ¿O hay animadversiones ideológicas?, o ambas cuestiones. Deberían explicarse claramente a los mexicanos las razones de esta postura, porque los demás países de nuestra región y de otras convergen en la idea de una asociación lo más estrecha posible con China. México desperdicia la tendencia histórica y su privilegiada situación geográfica, con un enorme litoral al Pacífico. ${ }^{6}$

\section{Contenido general y aspectos conflictivos y lesivos al interior del Acuerdo Transpacífico}

\section{Contenido esencial}

El cuerpo principal del Acuerdo incluye capítulos relativos a los programas de liberalización comercial, normas de origen, procedimientos aduaneros, defensa comercial o combate a las prácticas de abuso de posiciones dominantes, medidas sanitarias y fitosanitarias, barreras técnicas al comercio, lealtad comercial, propiedad intelectual, compras gubernamentales, comercio de servicios, transparencia, modalidades de cooperación bajo el título de “Asociación Estratégica” y sistema de solución de controversias. El Acuerdo principal es complementado por dos convenios más: un Acuerdo de Cooperación Ambiental y un Memorando de Entendimiento sobre Cooperación Laboral. Es necesario clarificar los términos de estos otros Acuerdos, conocerlos a detalle antes de que el Senado ratifique la incorporación de México.

\footnotetext{
${ }^{6}$ Navarrete, Jorge Eduardo: “El Siglo del Pacífico... ¿Americano?”. Copia del Original, Coordinaciòn del Programa Prospectiva Global, Centro de Investigaciones Interdisciplinarias en Ciencias y Humanidades, UNAM, 2012.
} 
Con respecto al comercio de bienes, el Acuerdo es muy ambicioso y de amplia cobertura. La gran mayoría de las posiciones arancelarias son objeto de inmediata y total desgravación. Los plazos máximos son de diez años, con excepción de los productos lácteos importados por Chile (quien logró pactar un Trato Especial ante la gran competitividad de Australia y Nueva Zelandia, por ejemplo), con respecto a los cuales y considerando su sensibilidad frente a las exportaciones neozelandesas, se fijó un período más extenso manteniendo los aranceles actuales durante los primeros seis años e iniciándose la desgravación sólo a partir de entonces para llegar a la desgravación total al cabo del décimo segundo año. Simultáneamente, se acordó una salvaguardia especial durante el período de transición, sobre la base de un mecanismo de activación automática.

Las principales expectativas, desde el punto de vista de los intereses de cualquiera de sus integrantes, fueron puestas en las acciones de cooperación y complementación con Nueva Zelanda para la investigación industrial, el desarrollo tecnológico y la capacitación profesional en áreas de especialización comunes a ambos países (sectores forestal y lácteo, entre otros). Con relación a Singapur, se esperaba un aprovechamiento de las ventajas ofrecidas por este país por su ubicación geográfica e infraestructura de obras y servicios. ${ }^{7}$ Los beneficios inmediatos que puede proporcionar el Acuerdo Estratégico de Asociación Transpacífico a México es que permitirá ingresar a mercados de dos continentes (exceptuando América, porque México ya tienen tLc con Estados Unidos y Canadá, Chile y Perú), como son Asia (a Vietnam, Singapur y Malasia) y Oceanía (Australia y Nueva Zelanda). Ello, inmediatamente en que entre en vigor legalmente el acuerdo.

Este acuerdo permitirá una mayor diversificación de la oferta exportable mexicana, siempre y cuando ésta esté disponible o sea posible de generar, y el impacto será muy benéfico, siempre y cuando el componente de valor sea mayoritariamente mexicano (no nacional, es distinto, en lo nacional participan empresas globales asentadas en México pero no propiedad de empresarios mexicanos) porque también se abre el mercado mexicano a nuevos flujos de exportaciones competitivas desde países como Australia o Nueva Zelanda y Malasia (los cuales tienen altos índices de consumo y oferta manufacturera y agrícola competitiva), Singapur y Vietnam, es decir, importaciones para México, que pueden desplazar a productores nacionales no competitivos, cuando sabemos que el flujo de importaciones a México ha

7 "Acuerdo de Asociación Transpacífico" (TPP), Sistema de Información sobre Comercio Exterior (SICE), OEA-SEDI-DDECT, http://www.sice.oas.org 
resultado lesivo y provocado un déficit comercial permanente, con la consecuente salida masiva de divisas. Asimismo, se podrán alcanzar beneficios relacionados al comercio de servicios, en este último rubro, México es también poco competitivo, ya que el déficit en servicios de la cuenta corriente, es la que más contribuye al déficit en cuenta corriente.

\section{Aspectos conflictivos y lesivos}

En las negociaciones desarrolladas ya entre los 10 países integrantes, Estados Unidos se ha empeñado en introducir fuertes medidas proteccionistas en el tema de la propiedad intelectual, aún más severas que las existentes en el Tratado de Libre Comercio entre Corea del Sur y EuA (que ha sido tomado como modelo de rigidez y proteccionismo al respecto), o en el llamado "Acuerdo Comercial Anti-Falsificación" (АСТА), e incluso, se ha comparado aquellas medidas promocionadas con el denunciado proyecto de "Ley-sopA" (Acta de Bloqueo a la Piratería en línea), y dentro de tales intenciones, se alerta, por ejemplo, ya que hay una solicitud concreta en ese sentido que afectaría la producción de medicamentos genéricos en países como el nuestro, de entrar en vigor con toda su normativa internacional estas disposiciones legales impulsadas por EUA. La idea de este último país podríamos resumirla diciendo que es un intento de protección absoluta, permanente y universal a la propiedad intelectual.

En suma, en el tema de la propiedad intelectual, no son solamente los aspectos de restricción en el uso de patentes (de producto y de proceso) y marcas cuyo plazo legal de exclusividad para el innovador ha vencido y pueden ser usadas las marcas y los procedimientos por otros productores, por ejemplo de medicamentos con su nombre genérico (el nombre de la sustancia o principio activo que contienen y no la marca del laboratorio que lo produce o comercializa), sino el proteccionismo comercial que ello implica y que violenta el principio de libre comercio que motiva los acuerdos de integración económica y comercial.

Otra cosa diferente es intentar bloquear legalmente la falsificación de productos y marcas, deseo y voluntad correcta y legítima para cualquier industrial innovador, porque la falsificación es un grave problema mundial que desalienta la innovación y hace perder por ese conducto mucho dinero a los industriales que dedican también cantidades importantes de sus ganancias a la innovación tecnológica, clave de la competitividad. Aquí, es fundamental diferenciar claramente lo uno de lo otro. Y ambas cuestiones se 
debaten dentro del Acuerdo Transpacífico, pero sin ser equivalentes en su perfil esencial y en sus efectos para sus distintos integrantes. Veamos con mayor detalle estos puntos.

\section{Propiedad intelectual y propuesta restrictiva y proteccionista de Estados Unidos}

Conforme a la tradición jurídica europea y latinoamericana, en la Declaración Mundial sobre la Propiedad Intelectual, votada en la Organización Mundial de la Propiedad Intelectual (OMPI), el 26 de junio del año 2000, la Propiedad Intelectual es entendida como "cualquier propiedad que, de común acuerdo, se considere de naturaleza intelectual y merecedora de protección, incluidas las invenciones científicas y tecnológicas, las producciones literarias o artísticas, las marcas y signos distintivos, los dibujos y modelos industriales y las indicaciones geográficas", es decir, como el goce y disfrute de un derecho patrimonial exclusivo reconocido sobre tales productos originales y técnicamente nuevos, o como mejoras a lo ya existente, o como diseños innovadores, otorgado por el Estado. En suma, tienen que ver con la capacidad creativa, y el titular del derecho tiene la facultad legal de evitar que otros tengan acceso o hagan uso de su propiedad sin su consentimiento.

En el caso de los productos informáticos, existe un movimiento social llamado "software libre" que considera que el término "Propiedad Intelectual" es engañoso y propone reunir bajo un mismo concepto diferentes regímenes jurídicos no equiparables entre sí, como las patentes, el derecho de autor, las marcas, las denominaciones de origen, entre otros, lo cual se aparta de la conceptualización y teorización internacionalmente reconocida. Otros críticos como Stephan Kinsella sostienen que los derechos de propiedad intelectual no son necesarios para promover la creatividad y el avance científico e imponen costos muy altos para la sociedad (incentivan costosos litigios judiciales, desincentivan la creación de mayor conocimiento una vez que el creador tiene el monopolio del derecho de propiedad intelectual).

La Propiedad Intelectual se clasifica en tres categorías (conforme a la OMPI):

1. Propiedad industrial o el derecho exclusivo que otorga el Estado para usar o explotar en forma industrial y comercial las invenciones o innovaciones de aplicación industrial o indicaciones comerciales que realizan individuos o empresas para distinguir sus productos o ser- 
Economía Informa núm. 380 mayo - junio • 2013 ㅁ

vicios ante la clientela en el mercado. Esta incluye las invenciones, marcas, patentes, dibujos y modelos industriales, así como indicaciones geográficas de origen.

2. Derechos de autor: El artículo 11 de la Ley Federal del Derecho del Autor define a los derechos de autor como el reconocimiento que hace el Estado a favor de todo creador de obras literarias y artísticas previstas en el artículo 13 de esta Ley, en virtud del cual otorga su protección para que el autor goce de prerrogativas y privilegios exclusivos de carácter personal y patrimonial. Los primeros integran el llamado derecho moral y los segundos, el patrimonial.

3. Derechos Conexos: comprende las interpretaciones o ejecuciones de los artistas, la producción de fonogramas y las actividades de los organismos de radiodifusión. ${ }^{8}$

Al término del tiempo de vigencia, el derecho de la patente (esta es la figura jurídica más conocida del sistema de propiedad industrial y la que más se utiliza para proteger el conocimiento que se genera en muchas de las áreas de investigación), o en caso de que no se hayan cumplido las cuotas anuales por la misma (en ocasiones, este es el requisito de vencimiento), se puede aprovechar la información contenida en ella sin el pago de las regalías correspondientes a los titulares de la patente. Es el caso de los llamados "medicamentos genéricos", entre muchos otros. La protección de las patentes es siempre un tema sumamente delicado en las negociaciones de tratados internacionales como los TLC y otros acuerdos (como el que nos ocupa), en donde, los países en vías de desarrollo que carecen de tecnología pretenden disminuir al mínimo la protección que se da a las patentes a fin de poder explotar dichos inventos a la brevedad posible. Mientras que, los países desarrollados pretenden extender al máximo el tiempo de protección con distintos mecanismos como las patentes de segundo uso, que son aquellas patentes que se conceden a invenciones ya patentadas pero a las cuales se les ha encontrado una nueva forma de uso o algún nuevo beneficio, y por consiguiente se concede un periodo adicional de protección.

Adicionalmente, existen otros temas de controversia como las licencias obligatorias que son aquellas que se otorgan a organismos públicos o a ter-

\footnotetext{
${ }^{8}$ Kinsella, Sthepan: "Contra la Propiedad Intelectual”, Documento en PDFwww.vanguardialibertaria.ne; y Solorio Pèrez Oscar Javier: "Derecho de la Propiedad Intelectual", http://www. indautor.gob.mx; y "Derechos de Propiedad Intelectual y el Acuerdo sobre los ADPIC", http:// www.wto.org/; también, "sopA: Hollywood's latest effort to turn back time”, 1 de noviembre de 2011,_http://news.cnet.com, “México firma ACTA; activistas rechazan su aplicación”, periódico El Economista, 11 de julio de 2012.
} 
ceras personas para la explotación de un invento sin el consentimiento del inventor en situaciones especiales como en emergencias nacionales, pero previa compensación al autor. Este tipo de licencias, también son discutidas en negociaciones de tratados, toda vez que para países con gran potencial inventivo este tipo de licencias son inadmisibles, mientras que en países pobres se procura siempre dejar esta alternativa. Son negociaciones muy difíciles en donde predomina regularmente el poder.

En México, la autoridad que se encarga de administrar todos estos derechos es la Secretaria de Economía a través del Instituto Mexicano de la Propiedad Intelectual (IMPI) y la ley que rige la materia es la Ley de Propiedad Industrial (LPI). El IMPI tiene como objetivo proteger sus derechos y promover y difundir los beneficios que ésta ofrece para apoyar la actividad inventiva y comercial de nuestro país, impulsando la creación y desarrollo de nuevas tecnologías en beneficio de toda la sociedad. La propiedad autoral o derechos de autor es administrada por la Secretaria de Educación Pública, a través del Instituto Nacional de Derecho de Autor.

El Capítulo 10 del Acuerdo Comercial que nos ocupa, que aborda el tema sobre la Propiedad Intelectual, se remite a los acuerdos respectivos registrados ante la omc y confirma los derechos y obligaciones al respecto de los firmantes o de cualquier otro acuerdo del que sean Partes, y agrega en el artículo 10.3 (“Disposiciones Generales") numeral 2:

Ninguna disposición de este Capítulo impedirá a una Parte adoptar las medidas necesarias para prevenir el abuso de los derechos de propiedad intelectual por parte de los titulares de los derechos o la utilización de prácticas que restrinjan injustificadamente, el comercio o que afecten, adversamente la transferencia internacional de tecnología, siempre que tales medidas sean consistentes con este Acuerdo. En particular, ninguna disposición de este Capítulo impedirá a una Parte adoptar las medidas necesarias para prevenir prácticas anticompetitivas que pudieran resultar del abuso de los derechos de propiedad intelectual. ${ }^{9}$

En la numeral 3 b) se indica restrictivamente, que las Partes se obligan también a: "establecer que las disposiciones de licencias no negociadas para productos, contenidas en formatos estándar, no impedirán a los consumidores ejercer las limitaciones y excepciones reconocidas en la legislación nacional de propiedad intelectual"; es decir, los integrantes del TPP quedan en libertad de tomar las medidas de protección necesarias evitando "prácticas

9 "Acuerdo Estratégico Transpacífico de Asociación Económica" traducción final, 12 de junio de 2005, www.sice.oas.or. 
anticompetitivas", siempre que no afecten el comercio ni la difusión tecnológica, y a restringir el uso de productos resguardados por los consumidores nacionales cuando las licencias no hayan sido negociadas. Es lo esencial.

Ahora bien, en materia de productos farmacéuticos, el planteamiento de EuA en la Ix Ronda de Negociaciones (celebrada en Lima Perú, del 19-28 de octubre de 2011) del Acuerdo de Asociación Transpacífico (TPP) donde participaron Australia, Brunéi Darussalam, Chile, Estados Unidos, Malasia, Nueva Zelanda, Perú, Singapur y Vietnam (México ausente), es en sustancia el de otorgar nuevos derechos a las grandes empresas farmacéuticas, en otras palabras, obtener nuevas patentes de explotación comercial por varias décadas (el promedio son 50 años para que venza la exclusividad) que implicaría varios efectos:

- los países negociadores con un mercado de medicamentos genéricos desarrollado (no se sabe exactamente cuáles, se incluye que toda la producción actual, que también varía de un país a otro), no tendrían la posibilidad de fabricar poder adquirir medicinas a menores costos utilizando referentes internacionales, perjudicando al sector más vulnerable de la población;

- las empresas farmacéuticas concentradas en dicho mercado, tendrían que concentrarse en otros como vía de sobrevivencia. Sería un grave trastorno para tales negocios, esencialmente de capital nacional, y la amenaza de cierre casi inmediato se cerniría sobre ellos;

- elevaría en el corto plazo, el costo de los medicamentos y por tanto encarecería el costo del cuidado de la salud, en este renglón.

- anula las disposiciones firmadas por los socios fundadores antes transcritas del Capítulo 10, artículo 10.3, numeral 2 que prohíbe "el abuso de los derechos de propiedad intelectual por parte de los titulares”, pero además, porque restringe el comercio internacional de tales productos. ${ }^{10}$

- El Senado de la República, deberá rechazar explícita y contundentemente este planteamiento y petición de los negociadores de Estados Unidos al respecto, mediante un Punto de Acuerdo que condicione a ello -entre otras cuestiones posibles- su eventual aprobación, por considerarlo violatorio de las propias disposiciones establecidas en el cuerpo de Acuerdo-TPP.

10 "Pedido de Estados Unidos en la Negociación del Acuerdo Transpacífico en materia de Medicamentos, hace peligrar disponibilidad de productos más baratos en nuestro país", Nota de Prensa No. 2, ix Ronda de Negociaciones del TPP, Lima Perú. Red GE (Globalización con Equidad), http://www.redge.org.pe 